\title{
Sterbehilfe vs. sterben lassen Vor die Entscheidung gestellt
}

Einsatzmeldung Atemnot, ein 45-jähriger Patient mit Lungenkarzinom im Endstadium. Ein nächtlicher Einsatz, der ganz anders ausgeht als erwartet und den erfahrenen Rettungsassistenten und Theologen Michael Frieß an seine persönliche Grenze bringt.

Verena Geywitz

Einsatzmeldung Es ist ein kühler Herbstabend im Oktober, draußen ist es dunkel und die Straßen sind fast leer. Michael Frieß und seine beiden Kollegen haben Nachtschicht. Seit knapp 4 Stunden sind sie jetzt auf der Rettungswache in Gröbenzell. Aber ausruhen können sie sich an diesem Abend nicht. Zwei Einsätze haben sie schon hinter sich, als die Leitstelle Fürstenfeldbruck eine neue Meldung durchgibt: Atemnot. Der Patient ist ein 45-jähriger Familienvater mit Lungenkarzinom im Endstadium. Ein Notarzt wird benötigt.

Abfahrbereit Der Ausrüstungscheck vor Schichtbeginn ist erfolgt: Für den Einsatz ist alles bereit. Innerhalb kürzester Zeit sind der 35-jährige Rettungsassistent und seine Kollegen startklar. Gegen 23 Uhr geht es mit Signal los in Richtung Emmering*. Der Ort liegt etwa 15 Minuten entfernt und gehört eigentlich gar nicht mehr zum Einsatzgebiet von Gröbenzell. Da der dortige Rettungswagen bei einem Einsatz ist, übernimmt die Rettungswache Gröbenzell den Einsatz. Die meisten Leute schlafen um diese Uhrzeit schon, es sind nur noch vereinzelt Autos auf den Straßen unterwegs. Die Fahrt verläuft ruhig. Frieß macht sich unterwegs noch keine Gedanken, was ihn am Einsatzort erwartet. Atemnot ist schließlich eine häufige Meldung.

Am Ziel angekommen Das Ziel ist ein 5-stöckiges Wohnhaus mitten im Zentrum 
von Emmering. Das Haus sieht unauffällig aus, ein ganz normales Mehrfamilienhaus. Im dritten Stock brennt noch Licht - da muss es sein. Schnell nehmen sie die Ausrüstung rennen zum Haus. Frieß klingelt an der Eingangstür. „Das Warten fühlte sich endlos lange an“, erinnert er sich. Als endlich der Türöffner summt, geht es im Eilschritt druchs Treppenhaus nach oben. Die komplette Ausrüstung muss mit, Aufzug gibt es keinen. Den Rettern ist sofort klar: Wenn der Patient in die Klinik muss, dann wird es ein mühsamer Transport durch das enge Treppenhaus.

Der erste Eindruck Eine etwa 40-jährige Frau öffnet die Wohnungstür. Sie stellt sich als Ehefrau des Patienten vor. Durch einen kurzen Flur geht es ins Wohnzimmer. Der erste Eindruck ist positiv: Der Patient ist ruhig und gefasst. Die Wohnung ist gutbürgerlich eingerichtet und es ist sehr ordentlich. Der 45-Jährige sitzt auf dem Sofa und ringt nach Luft, er ist blass, aber voll ansprechbar. Die beiden Kinder des Ehepaars schlafen schon und bekommen von der ganzen Aufregung nichts mit. Trotz der angespannten Situation hat die Familie alles im Griff. Frieß weiß noch genau, dass er darüber sehr froh war. Angsterfüllte und panisch reagierende Familienangehörige sind bei Einsätzen eine besondere Herausforderung.

Sauerstoffzufuhr verbessern Die ersten Maßnahmen sind klar: Zuerst einmal die Sauerstoffsättigung und das EKG überwachen und einen Zugang legen. Das Monitoring zeigt, dass der Patient dringend Sauerstoff benötigt. Er bekommt daraufhin $8 \mathrm{~L}$ Sauerstoff über eine Maske. Im Gespräch mit dem Patienten wird klar, dass er seine Diagnose sehr gut kennt. Er hat sich auf diese Situation bereits eingestellt.

Kühlen Kopf bewahren Trotz Sauerstoff verschlechtert sich der Zustand des Patienten. Alle warten auf den Notarzt. Die
Anspannung wächst und Frieß fühlt sich zunehmend unwohl. „Der Patient war inzwischen nicht mehr ganz so ruhig. Die zunehmende Luftnot und das Warten auf den Notarzt setzten ihm schwer zu“, erklärt Frieß. „Der Patient hatte Angst, seine Frau auch und trotzdem musste ich einen kühlen Kopf bewahren und den Patienten beruhigen.“

Hilflosigkeit Der Patient ist schwerstkrank und wird in den nächsten Tagen sterben. Das weiß er, das weiß auch seine Familie. Dem Patienten kann jetzt nur noch durch starke Schmerz- und Beruhigungsmittel geholfen werden. „Als Rettungsassistent ist das besonders schwer. Die benötigten Medikamente darf man nicht verabreichen. Es gibt keine Möglichkeit, die Situation erträglicher zu machen. Den Patienten zu beobachten, dabei zusehen zu müssen, wie sich die Lage verschlechtert, aber nichts tun zu können, das löst ein Gefühl von Hilflosigkeit aus.“

„Den Patienten zu beobachten, dabei zusehen zu müssen, wie sich die Lage verschlechtert, aber nichts tun zu können, das löst ein Gefühl von Hilflosigkeit aus."

Ein Hoffnungsschimmer Ein erleichtertes Aufatmen geht durch den Raum, als der Notarzt wenige Minuten nach dem Rettungsdienst eintrifft. Schnell wird die Tür geöffnet. Frieß erinnert sich genau an den hoffnungsvollen Blick des Patienten - endlich kann ihm jemand helfen. Der Notarzt ist zum Glück ein erfahrener Anästhesist. Frieß ist erleichtert und denkt: „Der ist Profi, der macht Schmerz- und Atemmedikamente als Hauptjob. Das ist genau der Richtige für diesen Einsatz.“
Fassungslos Der Notarzt verschafft sich einen ersten Überblick und untersucht den Patienten. Die Diagnose ist bekannt. Von Anfang an ist der Arzt sehr zurückhaltend, Medikamente gibt er keine. Er hört die Lunge ab und bespricht den aktuellen Zustand und die Erkrankung. Dann verschwindet er in der angrenzenden Küche. Frieß interpretiert: „Er konnte sich der Situation vermutlich nicht richtig stellen. Er hat sich ziemlich schnell in die Küche zurückgezogen, um dort das Protokoll auszufüllen.“

Panik im Wohnzimmer Zurück bleiben drei entgeisterte Rettungsdienstler, eine sprachlose Ehefrau und ein panischer Patient. Die Angst wirkt ansteckend. Die Frage steht im Raum: Was sollen wir jetzt tun? „In dem Moment schwappte die Panik des Patienten auf mich über, als ich merkte, der Notarzt macht nichts und die Situation eskaliert“, beschreibt Frieß seine Gefühle.

Luftnot Der Patient kann jetzt immer schlechter atmen. Er ist inzwischen kaltschweißig, sehr blass und ringt heftig nach Luft. Er ist unruhig und phobisch, dadurch verschlimmert sich sein Zustand noch weiter. Die Situation gerät immer mehr außer Kontrolle. Zahllose Fragen gehen Frieß durch den Kopf: Wieso tut der Notarzt nichts? Wie kann man dem Patienten helfen? Frieß berichtet, dass er immer wieder in die Küche gegangen ist und zum Notarzt gesagt hat: „Komm bitte rüber, der kriegt keine Luft!“ Von seinen Kollegen bekommt Frieß keine Unterstützung. Der Eine hat sich in seine Rolle als Fahrer zurückgezogen, der zweite Kollege ist noch sehr unerfahren und bleibt im Hintergrund.

Ausnahmezustand Die Situation ist für alle Beteiligten unerträglich, vor allem für den Patienten. Er ahnt, wie es um ihn steht und seine Panik verschlimmert die ganze Situation. „Wenn es so weiter geht, dann erstickt er hier im Wohnzimmer", denkt 
Frieß. Er hält es nicht mehr aus und läuft aufgebracht in die Küche. Angestaut haben sich Wut und Empörung, er kann nicht mehr untätig daneben stehen. Als er den Arzt beim Ausfüllen des Protokolls antrifft, platzt Frieß der Kragen. Obwohl er den Notarzt zur Rede stellt und ihn versucht davon zu überzeugen, doch noch Medikamente zu geben, bleibt der Arzt stur. Keine Medikamente, der Entschluss steht fest. Der Patient soll schnellstmöglich ins nächstgelegene Krankenhaus gebracht werden.

„Als Rettungsassistent habe ich hier meinen Verantwortungsbereich überschritten. Es steht mir ja nicht zu, dem Arzt in dieser Situation klare Vorschläge zu machen.“

Verantwortungsbereiche „Als Rettungsassistent habe ich hier meinen Verantwortungsbereich überschritten“, gibt Frieß zu. „Es steht mir ja nicht zu, dem Arzt in dieser Situation klare Vorschläge zu machen.“ Rückblickend erzählt er: „Es war dann auch im Team nicht mehr gut. Ich habe mich überfordert gefühlt, weil der Patient mir unter den Händen wegstarb. Und zwar unter Qualen. Ich traf da beim Arzt aber auf kein Verständnis und kein Gehör.“

Transport ins Krankenhaus Der Patient wird zum Transport vorbereitet und auf einen Tragestuhl gesetzt. Das ist gar nicht so einfach. Er ist inzwischen völlig aufgelöst und lässt sich nicht mehr beruhigen. Das enge Treppenhaus wird zur Herausforderung. Mit jedem Treppenabsatz eskaliert die Situation ein bisschen mehr. Frieß erinnert sich, dass es ein grauenhaftes Gefühl war, einen erstickenden Menschen durch ein Treppenhaus zu tragen.
Ein letzter Versuch Als er die Situation nicht mehr aushält, läuft Frieß nochmals zum Arzt, der noch in der Wohnung ist. Ein letzter Versuch, doch noch eine Medikamentengabe zu erwirken - leider erfolglos. Die Anweisung gilt: Der Patient soll schnell zum Wagen und dann ins nächste Krankenhaus gebracht werden. Frieß stürmt zum Rettungswagen zurück und hilft seinen Kollegen. Beim Umlagern auf die Trage wird der Patient bewusstlos.

Verabschiedung Die Frau kann sich nicht mehr von ihrem Mann verabschieden. Sie ist sehr gefasst, auch jetzt noch. Im Krankenwagen fährt sie aber nicht mit, sie bleibt bei den beiden Kindern zurück. Abgesprochen ist, dass keine Reanimation durchgeführt wird, sollte es zum Herzstillstand kommen.

Im Rettungswagen Wenige Minuten später stirbt der Patient im Krankenwagen. Die Situation hat Frieß noch klar vor Augen: Herzstillstand - der EKG-Monitor zeigt noch ein paar breite QRS-Komplexe, dann eine Nulllinie. Der Patient wird noch ins Krankenhaus gebracht. In einem kahlen gekachelten Raum findet die Übergabe statt. Viel gibt es nicht mehr zu sagen.

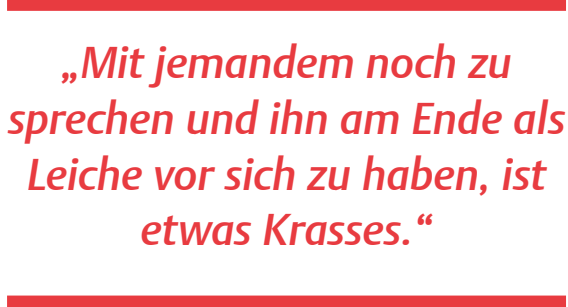

Nachbesprechung Im Gespräch mit dem Notarzt versucht Frieß herauszufinden, warum dieser Einsatz so eskalierte. An die Antwort des Arztes - eine halbe Erklärung für sein Verhalten - erinnert sich Frieß noch heute genau: „Ich konnte den doch nicht umbringen." Mehr sagt der
Arzt nicht. Die Angst davor, dass der Patient noch schneller stirbt, wenn er Beruhigungs- oder Schmerzmittel bekommt, hat ihn an der Medikamentengabe gehindert. Er wollte auf keinen Fall durch Medikamente einen aktiven Beitrag zum Tod des Patienten leisten.

Rückblick Natürlich kommt es vor, dass Patienten versterben, das ist auch Frieß klar. Dieses Mal war es aber anders, erinnert er sich: „Mit jemandem noch zu sprechen und ihn am Ende als Leiche vor sich zu haben, ist etwas Krasses." Aber auch das Wissen, dass dem Patient hätte geholfen werden können, macht es besonders schwierig. Er hätte starke Schmerzmittel, aber vor allem auch Beruhigungsmittel gebraucht. Dann hätte er ruhiger einschlafen können. So ist der Patient unnötig qualvoll gestorben. „Das war wirklich ein ganz schlechter Einsatz."

Beitrag online zu finden unter http://dx.doi.org/ $10.1055 / \mathrm{s}-0042-117267$

\section{Spannende Einsätze}

Hatten auch Sie einen außergewöhnlichen Einsatz? Ob positiv oder negativ - in retten! können Sie davon erzählen und Ihre Kollegen am konkreten Beispiel lernen lassen. Sie erreichen die Redaktion unter 0711/8931-652 oder unter retten@thieme.de. 


\section{Palliativmedizin im} Rettungsdienst Wann werde ich zum Mörder?

\section{Ein Kommentar von Dr. Sönke Müller}

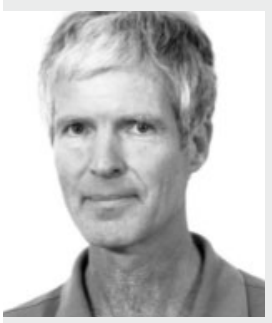

Eid des Hippokrates „Ich werde niemandem, auch nicht auf seine Bitte hin, ein tödliches Gift verabreichen oder auch nur dazu raten...“ (ca. 400 v. Chr.).

Der Notarzt war unmenschlich! Ich konnte es zunächst fast nicht glauben, als ich den Einsatzbericht zu lesen bekam. Was für ein Versager war denn hier am Werk gewesen? „Beruf verfehlt!“, „Herzund gefühllos!“, „Keine Ahnung von Menschlichkeit!“, „Wohl noch nie was von Palliativmedizin gehört!“, „Der sollte seinen Notarztschein oder die Approbation am besten umgehend zurückgeben!“ - das waren spontan meine ersten „unkontrollierten“ Gedanken.

Furcht vor den Folgen Bei genauerem Nachdenken und nach nochmaligem Lesen der Fallbeschreibung spürte ich dann aber doch, was den Notarzt zu seiner - im Sinne einer leidensbegrenzenden Maßnahme - passiven Haltung bewogen hat: Angst. Angst davor, als direkte Konsequenz einer Maßnahme ein Leben zu beenden: „Ich setze die Spritze an, der Patient macht noch ein paar Atemzüge und verstirbt als ganz logische Folge meiner z.B. atemantriebsmindernden Schmerztherapie. Ich habe ein Leben beendet! Völlig konträr zu dem Beweggrund, aus dem ich Arzt geworden bin, und auch dem Eid des Hippokrates (s. oben). Ich habe mich zum Herr über das Leben erhoben und einen eigentlich mitten im Leben stehenden Familienvater in den Tod befördert. Da nützt es mir wenig, wenn ich von der schweren Erkrankung des Patienten erfahren habe. Eben hat er noch gelebt, jetzt ist er unwiderruflich tot, „vermittelt“ durch mein Handeln. Dieser Gedanke wird mich verfolgen - ob ich das aushalte? Da ziehe ich mich doch besser zum Protokollschreiben in die Küche zurück...“

Oder war die Menschlichkeit doch zu groß? Vielleicht war der Notarzt in dem geschilderten Fall gar nicht unfähig, sondern im Gegenteil als Arzt zu menschlich, zu wenig hart und zu wenig in sich ruhend, um den Tod eines Menschens als Erlösung mit in Kauf zu nehmen. Der um Luft ringende Patient hat freilich von dieser Form der Menschlichkeit nichts; ihm wird stattdessen die Möglichkeit eines würdevollen Sterbens genommen.

Gibt es eine Lösung? Da hilft vielleicht nur eins: Notärzte und Rettungsdienstler auf solche Situationen vorbereiten - medizinisch, rechtlich, ethisch und psychologisch. Es gibt noch viel zu tun!

Dr. Sönke Müller ist als hausärztlicher Internist in Neckargemünd niedergelassen. Neben seiner Praxistätigkeit arbeitet er als Notarzt im regionalen Rettungsdienst. Er ist Mitherausgeber von retten!. E-Mail: Soenke.Mueller@t-online.de 